\title{
Large tuning in the electrowetting behaviour on ferroelectric PVDF-HFP/Teflon AF bilayer
}

\author{
Sandip M. Wadhai ${ }^{1}$, Yogesh B. Sawane ${ }^{2}$, Abhay. V. Limaye ${ }^{1}$, and Arun G. Banpurkar ${ }^{1, *}$ (1) \\ ${ }^{1}$ Department of Physics, Centre for Advanced Studies in Materials Science and Condensed Matter Physics, Savitribai Phule Pune \\ University, Pune 411007, India \\ ${ }^{2}$ Department of Physics, Ahmednagar College, Ahmednagar 414 001, India
}

Received: 16 March 2021

Accepted: 30 June 2021

Published online:

12 July 2021

(C) The Author(s), under exclusive licence to Springer Science+Business Media, LLC, part of Springer Nature 2021

\begin{abstract}
Electrowetting (EW) response on a dielectric depends on its permittivity value, Young contact angle and voltage amplitude. We present a large change in EW contact angle, from $163^{\circ}$ to $80^{\circ}$, on the bilayer dielectric made up of ferroelectric PVDF-HFP with a thin layer of fluoropolymer. The thickness values of both layers were separately optimized for high effective capacitance essential for the large EW response. It reveals that the bilayer with $\sim 500 \mathrm{~nm}$ thick PVDF-HFP layer and $\sim 50 \mathrm{~nm}$ thin layer of Teflon results in the maximum value of effective dielectric constant, $\varepsilon \approx 8$. Besides this gain, dc-voltage EW response exhibits hysteresis mainly due to polarization in the ferroelectric layer such that, hysteretic offset voltage was found to depend on the applied voltage amplitude and thickness of the dielectrics. Finally, bilayer was subjected to ac-voltage EW in silicone oil for ambient temperature ranging from -25 to $70{ }^{\circ} \mathrm{C}$. The consistent EW response in this ambient without any degradation/delamination of polymer surface confirmed the durability of the bilayer on the transparent ITO electrodes.
\end{abstract}

\section{Introduction}

Electrowetting on dielectric (EWOD or EW) is one of the most versatile tools in controlling the wettability of conductive liquid drop on a dielectric surface; therefore, it is implemented in many microfluidic devices [1, 2]. Generally, amorphous fluoropolymers are used as a dielectric coating on a planar electrode in many of these applications like optical lens component $[3,4]$, video displays $[5,6]$, beam steering devices [7], lab-on-a-chip devices [8], digital microfluidics $[9,10]$, biomedical applications [11] etc.

There is a growing demand for digital microfluidics point of care devices for various diagnoses of diseases including COVID-19 [12]. Therefore, the

Handling Editor: Chris Cornelius.

Address correspondence to E-mail: agb@physics.unipune.ac.in 
expert scientific laboratories are engaged in the development of common and affordable EWOD platforms suitable to all public settings. In this direction, cloud-based open source EWOD cyber manufacturing ecosystem is proposed by CJ Kim et al. [13]. This enables an automatic translation from user requirements to manufactured digital microfluidics. M. Ibrahim and K. Chakarabarty have introduced a new paradigm for biochip design and adoption of biochip for running biomolecular protocols [14]. The EWOD hardware includes the electrode design, their interconnection to the control electronics and dielectric coating on these electrodes. The reduction in the driving voltage in EWOD by improving the dielectric property is an open-ended issue after the inception of first EWOD device by Bruno Berge [15]. Since then, various attempts were made in designing dielectrics and bilayers with inorganic and organic layers.

The dielectric was usually made from Teflon AF [16], Cytop [17] and parylene [18]. This unique choice is due to their excellent properties like optical transparency, chemical stability, negligible contact angle hysteresis and high Young contact angle. However, the dielectric constant of these polymers is about $\varepsilon \sim 2$, thus high driving voltage, typically $\sim 100 \mathrm{~V}$ is required to achieve desired change in contact angle. The EW is a versatile tool that offers precise and reversible control on the wettability of a liquid droplet on the dielectric surface by applying external voltage [2]. The increase in wetting, i.e. apparent reduction in contact angle $\theta(U)$ upon application of external voltage amplitude, is given by Young-Lippmann electro-capillarity equation [19];

$\cos \theta(U)=\cos \theta_{Y}+\eta$

Here, $\theta(U)$ is voltage-dependent contact angle, $\theta_{Y}$ is Young contact angle and $\eta=\varepsilon \varepsilon_{0} U^{2} / 2 d \gamma_{L V}$ is the dimensionless electrowetting number. It is the ratio of electrostatic energy per unit area of parallel plate capacitor and surface energy per unit area of that droplet with respect to the surrounding medium. The dielectric capacitance per unit area is given by $C=\epsilon \epsilon_{0} / d$, and it is proportional, directly to dielectric constant $\varepsilon$ and inversely to dielectric thickness d. Large tuning in EW response starting from Young contact angle can be realized for relatively low voltage on insulators with high dielectric constant. In principle, high capacitance can be attained by using very thin dielectric but early dielectric breakdown puts a constraint on the lower limit of dielectric thickness. This stimulates the use of newer insulators with both properties like high dielectric constant and low surface energy.

Most of the researchers are making efforts to improve the dielectric constant by utilizing two or more layers of dielectric. Typically, composite metaloxide or nitrides dielectrics are used to increase the effective dielectric constant. Recently, Wang et al. fabricated $\mathrm{La}_{2} \mathrm{O}_{3}$ on titanium substrate to observe superlow voltage electrowetting [20]. Khan et al. have reported the performance of $\mathrm{SiO}_{2}$ as a dielectric material for beam steering electrowetting devices [21]. Homogeneous dielectric structure of $\mathrm{ZrO}_{2} / \mathrm{Te}-$ flon nanocomposite with high breakdown field is utilized for electrowetting applications [22]. The dielectric materials with high permittivity like Cytop$\mathrm{SiO}_{2}$ bilayer [23], anodic $\mathrm{Ta}_{2} \mathrm{O}_{5}$ with a top layer of fluoropolymer [24], $\mathrm{La}_{2} \mathrm{O}_{3}$ on anodic aluminium oxide (AAO) [25], nanocomposite thin film of barium strontium titanate (BST)/Teflon [26], plasma-enhanced chemical vapour deposition (PECVD) of fluorocarbon [27] and nanocomposite dispersion of $\mathrm{BaTiO}_{3}[28]$ were used for electrowetting studies. The synthesis of these oxides requires specialized techniques like chemical vapour deposition, plasma deposition, sputtering, atomic layer deposition and electrochemical anodization. Also, large area deposition of many inorganic dielectrics is not cost effective. On the other side, thermoplastic fluoropolymer poly(vinylidene fluoride-co-hexafluoropropylene)(PVDF-HFP) is an inert and widely known for good electrical insulation with high ferroelectric polarization $[29,30]$ yet it is less explored in the EWOD applications.

The PVDF-HFP polymer possesses a high dielectric constant $(\varepsilon \sim 10)$, but earlier result reveals poor EW contact angle recovery when voltage is switched off. Therefore, additional top layer of Teflon like amorphous fluoropolymer is necessary to overcome this obstacle. The introduction of a top coating on a ferroelectric layer reduces the effective dielectric constant of the bilayer. This aspect involves careful selection of the parameters like thickness for the optimal EW output. Recently, Sawane et al. have studied electrowetting response on PVDF-HFP insulators and verified that it is suitable dielectrics for electrowetting application with effective dielectric constant, $\epsilon_{\text {eff }} \approx 6$ [31]. In similar study, this bilayer is 
utilized for the ac and dc voltage EW for examining the role of ac frequency on EW response. The issue of charge trapping in this bilayer dielectric for dc voltages is also briefly discussed [32]. The dielectric constant for PVDF-HFP at low frequency is about $\varepsilon \approx$ 10 and that of Teflon $\mathrm{AF}$ is about $\varepsilon \approx 1.98$. Thus, variation in thickness of both layers is experimentally accessible parameter-space to achieve highest capacitance on this bilayer dielectric. Papathanasiou and his group experimentally and computationally supported that the effect of dielectric thickness and elasticity on the onset of the saturation [33]. The saturation in the EW contact angle remains an unresolved issue with several supportive arguments without unified consensus [34].

In the present study, we provide the results utilizing ferroelectric-Teflon AF bilayer for maximum EW contact angle change. Therefore, dielectric thickness is optimized for both PVDF-HFP and Teflon AF layers to realise large tuning in contact angle at low voltage amplitude. The dielectrics, PVDF-HFP and Teflon AF thickness values were varied from nano-metre to micro-metre followed by careful estimation of the effective dielectric constant from ac-voltage electrowetting response. We further examined the dc-voltage electrowetting response to investigate the probable ferroelectric contribution in the EW response.

\section{Materials and methods}

\section{Dielectric film deposition and characterization}

The polymer PVDF-HFP granules (Aldrich, average Mol wt. 4,00,000) and acetone (SigmaAldrich, $\geq 99.9 \%$ ) were used for preparation of a coating solution. The solution of PVDF-HFP was prepared in acetone by constant stirring. The solution was filtered through PTFE syringe filter of average pore size of $\sim 1 \mu \mathrm{m}$. The PVDF-HFP insulator films were deposited on indium tin oxide (ITO) glass substrates by dip-coating technique. The ITO glass substrates (Aldrich, USA) were cleaned using diluted detergent solution prior to the deposition. The detergent traces were removed by rinsing the substrates in deionized water several times. Further, the substrates were ultrasonically cleaned using acetone, ethanol and isopropanol to remove the traces of the detergent. The substrates were dried in nitrogen flow and stored in a laminar flow cabinet. To study the effect of the thickness of ferroelectric layer on ac and dc-voltage electrowetting, PVDF-HFP with various thickness values was deposited on separate ITO glasses. The thickness was controlled by varying parameters like polymer concentrations in acetone (solution viscosity), withdrawal speeds and the number of dip-coating layers. Typically, concentration of PVDF-HFP in acetone was varied from 4 to 12 wt $\%$. The substrate retracting speed during dipcoating was kept at $1 \mathrm{~cm} / \mathrm{min}$ and $7 \mathrm{~cm} / \mathrm{min}$. The number of layers was varied from 1 to 5 for the desired thickness of PVDF-HFP. These films were dried in laminar flow and cured in a vacuum oven at $100{ }^{\circ} \mathrm{C}$ for $2 \mathrm{~h}$ for the complete removal of the solvent. The film thickness was estimated using optical profilometer, and representative experiment is shown in electronic supplementary information ES1(a). The measurement reveals PVDF-HFP thickness ranging from $190 \pm 10 \mathrm{~nm}$ to $7.7 \pm 0.01 \mu \mathrm{m}$. Finally, a thin layer of Teflon AF (The Chemours Company, USA) was dip-coated on each substrate by using Teflon AF solution (1 wt \%, in Fluorinert FC 40, Sigma-AldrichUSA) with withdrawal speed of $1 \mathrm{~cm} / \mathrm{min}$. Such substrates were finally cured in vacuum oven at $100{ }^{\circ} \mathrm{C}$ for 2 to $3 \mathrm{~h}$ for complete removal of the fluoropolymer solvent. The three sets of such bilayer films were prepared for three different Teflon thickness viz. 50, 180 and $320 \mathrm{~nm}$.

PVDF-HFP film was subjected to the X-ray diffraction (XRD) study after the final heat treatment. The PVDF-HFP film of thickness $2.23 \mu \mathrm{m}$ was characterized using X-ray radiation, $\mathrm{Cu} K_{\alpha}=1.542 \AA$ immerging from nickel filter. Figure ES1 (b) shows the plot of XRD intensity against $2 \theta$ value. The XRD spectrum for PVDF-HFP shows partial crystalline nature. The peak at $2 \theta=20.7^{\circ}$ corresponds to $\beta$ phase of PVDF-HFP. This $\beta$ phase offers the ferroelectric nature to the PVDF-HFP polymer. The poor crystallization of PVDF-HFP in different phases leads to weak ferroelectric signature.

The surface morphologies of PVDF-HFP and PVDF-HFP/Teflon bilayer are examined using AFM and FESEM techniques. Figure ES1 (c) and (d) shows AFM images of PVDF-HFP film and PVDF-HFP/ Teflon bilayer, respectively. Figure ES1(e) shows FESEM image of the PVDF-HFP surface with the thickness of $2.23 \mu \mathrm{m}$. The AFM morphology clearly shows that the surface roughness for PVDF-HFP film 
is higher in comparison with surface roughness after Teflon AF layer. The smooth surface is essential for minimal contact angle hysteresis and contact angle recovery in EW process.

\section{Electrowetting}

The electrical contacts were made on the bottom ITO electrode by indium metal soldering. All the EW experiments were carried out in silicone oil (viscosity $\mu=5 \mathrm{cSt}, \rho=0.971 \mathrm{gm} / \mathrm{cc}$, Sigma-Aldrich). The oil ambient reduces gravity effects on EW; additionally thin lubricating oil layer under the droplet reduces contact angle hysteresis. The ac and dc-voltage electrowetting responses were extensively tested on each substrate in silicone oil ambient in the quartz cuvette $\left(40 \times 40 \times 40 \mathrm{~mm}^{3}\right)$. The schematic diagram of EW setup is shown in Fig. 1 (bottom inset). The conductivity of deionised water was increased to $0.5 \mathrm{mS}(\mathrm{cm})^{-1}$ by adding $0.01 \mathrm{M} \mathrm{KCl}$ and used as a drop phase in all EW experiments. The droplet volume was kept at $6 \mu \mathrm{l}$. A function generator (Agilent 33220A) was used to generate ac and ramped (pseudo dc voltage) signal and further amplified to the preferred value using a custom-built high voltage OP-amplifier. The voltage was applied between aqueous droplet and the bottom ITO electrode, usually at ground potential. We have repeated the EW response for several voltage cycles and simultaneously recorded contact angle, $\theta(U)$ using optical contact angle (OCA-15 + DataPhysics Instruments,

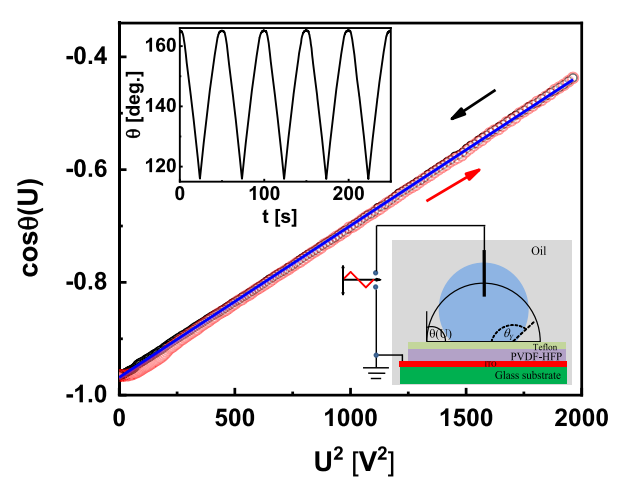

Figure 1 Plot of EW response on PVDF-HFP $(1.68 \mu \mathrm{m}) /$ Teflon $\mathrm{AF}(50 \mathrm{~nm})$ bilayer as a function of square of ac-voltage amplitude. The increasing voltage cycle is shown by red arrow and black arrow depicts decreasing voltage cycle. The solid blue line shows least square-linear fit to the EW data points. Top inset shows EW response $\theta(U)$ as a function of time advance. The bottom right inset represents the schematic of EW setup.
Germany) goniometer at $50 \mathrm{fps}$ and analysed using SCA-20 software (DataPhysics Instruments, Germany).

\section{Result and discussion}

The EW response on PVDF-HFP/Teflon AF bilayer dielectrics with a range of thickness values is studied for ac and dc-voltages. Figure 1 shows the plot of EW response in terms of cosine of contact angle as a function of the square of ac-voltage amplitude. The EW response starts from Young contact angle $\left(\theta_{\mathrm{Y}}=\right.$ $163^{\circ} \pm 1^{\circ}$ ) following identical paths for increasing and decreasing voltage cycles. This clearly demonstrates reversible EW contact angle change with negligible contact angle hysteresis (see Fig. 1, top inset).

Three different sets of bilayer films were tested for several ac-voltage cycles, and one representative experiment output is shown in Fig. 1. In each of the sets, PVDF-HFP thickness was varied from few tens of nano-metres to a few micrometres, while Teflon AF thickness was kept fixed. The EW response in terms of $\Delta \cos \theta=\cos \theta(U)-\cos \theta_{\Upsilon}$ versus voltage square $\left(U^{2}\right)$ is plotted against different PVDF-HFP thickness for Teflon thickness $d_{T}=50 \mathrm{~nm}$ (See Fig. 2 inset). The maximum voltage amplitude was maintained below the saturation regime. Notably, linear behaviour following Young-Lippmann (Eq. 1) is observed for all polymer thickness. The graph clearly indicates the increase in the slope of EW response with decreasing thickness of PVDF-HFP layer. The value of the slope of each plot is further utilized to estimate effective capacitance per unit area of the bilayer dielectric using liquid-oil surface tension $\gamma_{L O}=38 \mathrm{mN} / \mathrm{m}$ as an input parameter [35].

Figure 2 shows a plot of equivalent capacitance of bilayer estimated from EW response for different combination of the thickness values. The plot indicates that the experimental values of equivalent capacitance per unit area $C_{\mathrm{eq}}$ are in close agreement with the theoretical model [36].

$$
C_{e q}=\frac{\varepsilon_{0} \varepsilon_{T} \varepsilon_{P}}{\varepsilon_{T} d_{P}+\varepsilon_{P} d_{T}}
$$

Here, $\varepsilon_{p}$ and $\varepsilon_{T}$ are dielectric constants of PVDFHFP and Teflon AF layers, respectively. Also, $d_{P}$ and $d_{T}$ are thickness of PVDF-HFP and Teflon AF layers, respectively. Using this formula, we have estimated 


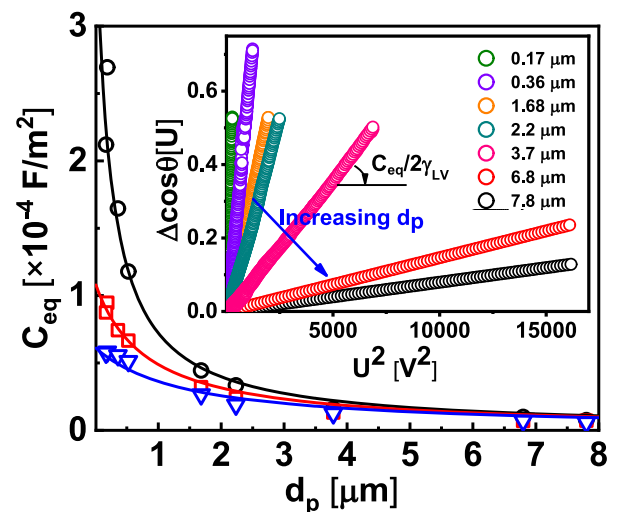

Figure 2 The plot of equivalent capacitance for varying thickness of PVDF-HFP and for three values of Teflon AF thickness (O$50 \mathrm{~nm}, \square-180 \mathrm{~nm}$ and $\nabla-320 \mathrm{~nm}$ ). The solid lines indicate the theoretical curve of equivalent capacitance per unit area evaluated using Eq. 2 for combination PVDF-HFP and Teflon AF thickness. Inset shows plot of $\Delta \cos \theta(U)$ against square of ac-voltage on bilayers varying PVDF-HFP thickness and constant Teflon thickness $d_{T}=50 \mathrm{~nm}$.

the equivalent capacitance for all the thickness combinations of the bilayer and it is shown by solid lines. The data points observed in our experiment are in close agreement with theoretical curve further validating the model. A significant increase in capacitance can be noted when the thickness of PVDF-HFP is below $0.7 \mu \mathrm{m}$ with $d_{T}=50 \mathrm{~nm}$. A very thin, tens of $\mathrm{nm}$ thin dielectric layers are omitted from the study due to the leakage current and early dielectric breakdown. Therefore, trade-off is necessary in deciding the optimum value of bilayer thickness for the desired EW response. The graph further depicts that the equivalent capacitance decreases with increasing thickness of both PVDF-HFP and Teflon AF layer. In particular, for $d p>7 \mu \mathrm{m}$, all plot reaches to a constant value evident from to the inverse proportionality of the dielectric thickness (see Eq. 2). The equivalent capacitance $\left(C_{e q}\right)$ for $d p>7 \mu \mathrm{m}$ reaching towards lowest value which is less useful for practical purpose.

The above EW data are further utilized for determining the effective dielectric constant of PVDFHFP/Teflon AF bilayer. The equivalent capacitance for this bilayer dielectric, $C_{e q}$ can be further expressed as:

$C_{e q}=\varepsilon_{0} \varepsilon_{e f f} / d$

Here, $\varepsilon_{e f f}$ is the effective dielectric constant of the bilayer and $d$ is the total thickness of the bilayer. We observed the maximum value of effective dielectric constant of $\varepsilon_{e f f} \approx 8$ for PVDF-HFP thickness $d_{p \text { - }}$ $\sim 500 \mathrm{~nm}$ and Teflon AF thickness of $d_{T} \sim 50 \mathrm{~nm}$. The EW response on such bilayer is efficient, i.e. maximum EW response for a given input voltage.

We further quantify the efficacy of EW response by analysing absolute change in slope value, i.e. $\left(\left|\frac{\Delta \theta}{\Delta U}\right|\right)$. Here, $\Delta \theta=155^{0}-130^{\circ}=25^{0}$ is chosen for estimating this slope value (see Fig. 3 , inset). The choice of this region is due to the linearity in $\theta(U)$ variation against $U$. The absolute slope value is calculated for all the above combinations of $d_{P}$ and $d_{T}$ values. Figure 3 shows the plot for absolute value of $\left(\frac{\Delta \theta}{\Delta U}\right)$ against the insulator thickness $\left(d_{P}\right)$ values ranging from 0.2 to $8 \mu \mathrm{m}$.

This plot clearly indicates that absolute value of $\frac{\Delta \theta}{\Delta U}$ monotonically increases as PVDF-HFP thickness decreases except the thickness value $d_{\mathrm{P}}$ less than 0 . $4 \mu \mathrm{m}$. Notably, highest value of $\left|\frac{\Delta \theta}{\Delta U}\right|$ is observed for $d_{\mathrm{P}}$ in the range $0.4 \mu \mathrm{m} \leq d_{P} \leq 0.7 \mu \mathrm{m}$ and $d_{\mathrm{T}}=50 \mathrm{~nm}$. This regime of thickness combination shows maximum EW response for this bilayer. The decrease in $\left|\frac{\Delta \theta}{\Delta U}\right|$ for PVDF-HFP thickness, $d_{p}<0.4 \mu \mathrm{m}$ can be explained from behaviour of plot of successful EW regimes for PVDF-HFP and Teflon AF [31]. The low dielectric thickness usually yields higher value of capacitance, affecting the EW response. But when insulator (PVDF-HFP) thickness is few tens of nanometre, the leakage current through dielectric waning charge density at the interface [31]. This emphasizes choice of thickness combination of ferroelectric and

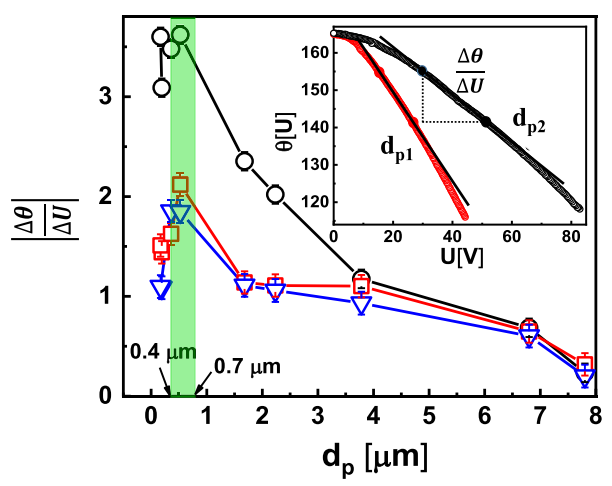

Figure 3 The absolute change $(\Delta \theta / \Delta U)$ is plotted against PVDF-HFP thickness and for three values of Teflon AF thickness ( O-50 nm, $\square-180 \mathrm{~nm}$ and $\nabla-320 \mathrm{~nm}$ ). Inset shows EW response on bilayer dielectrics with $d_{P 1}=1.7 \mu \mathrm{m}, d_{p 2}=$ $6.8 \mu \mathrm{m}$ and constant $d_{T}=50 \mathrm{~nm}$. The solid black line fit indicates change in contact angle per applied voltage $\left(\frac{\Delta \theta}{\Delta U}\right)$. 
hydrophobic polymer is very crucial for the effective change in EW contact angle on every dielectric including bilayer and multilayer dielectrics.

Further, this bilayer dielectric is tested for dcvoltage EW shown in Fig. 4a. It shows that EW contact angle decreases right from positive voltage bias, i.e. $U=0$ to $U_{\max }$. But it does not follow an identical path while reducing the voltage amplitude. Notably, EW contact angle remains pinned till the certain voltage value, termed as offset voltage $(\Delta U)$. The same behaviour is also seen for the negative voltage cycle. The contact angle modulation for dc-voltage is mainly affected by polarization-dependent contact angle hysteresis. The representative bilayer having a thickness of PVDF-HFP as $d_{P}=2.23 \mu \mathrm{m}$ and that of Teflon $\mathrm{AF} d_{T}=50 \mathrm{~nm}$ was utilized for the study. The effective dielectric constant for the film is $8.01 \pm 0.05$. We claimed that the unwanted hysteresis in dc-EW is due to the ferroelectric property of PVDF-HFP layer that gives the maximum offset voltage of $\Delta U \approx 17 V$ [31]. The XRD studies indicate the emergence of ferroelectric $\beta$ phase of the PVDF-HFP. The detailed study, like effect of polymer thickness, applied voltage on the hysteresis is presented in subsequent section which is missing in the previous reports [31, 32].

The inset graph of Fig. 4a shows that the offset voltage increases with the maximum level of applied voltage $\left(U_{\max }\right)$. However, it does not increase further although maximum input voltage increases towards saturation contact angle.

We further investigate the dependency of the hysteretic behaviour on the dielectric thickness. Figure $4 \mathrm{~b}$ shows variation of saturated offset voltage,

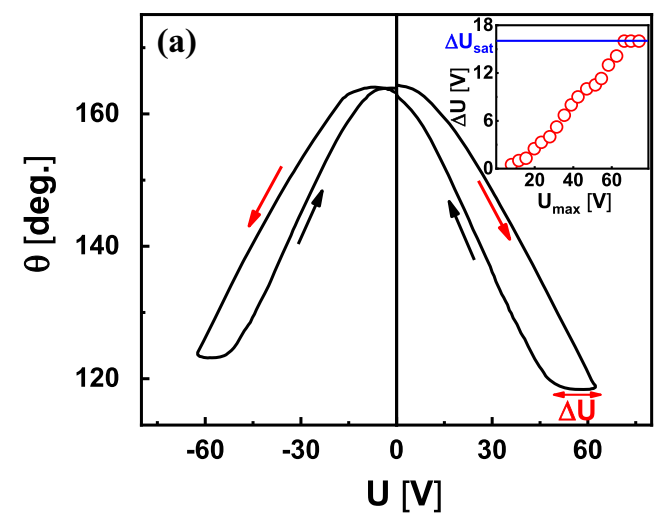

Figure 4 a EW response for dc-voltage on a bilayer $\left(d_{P}=2.23 \mu \mathrm{m}\right.$ and $\left.d_{T}=50 \mathrm{~nm}\right)$. The arrows, red and black, indicate the increasing and decreasing voltage, respectively. Inset
$\Delta U_{\text {sat }}$ with increasing PVDF-HFP thicknesses and for three values of Teflon AF thickness. It shows that $\Delta U_{\text {sat }}$ also increases with PVDF-HFP thickness and saturation in this value at higher PVDF-HFP thickness is observed. The ferroelectricity in PVDF-HFP layer is limited by the intrinsic polarization and the segregation of $\alpha$ and $\beta$ phases in the PVDF-HFP layer. Usually, $\beta$ phase appears for higher film thickness and with suitable process parameters. This phase contributes to high value of polarization [37]. As shown before, with increasing thickness value of $d_{\mathrm{P}}$, the equivalent capacitance saturates to its lower limit $C_{e q} \sim 0.2 \times 10^{-4} \mathrm{~F} / \mathrm{m}^{2}$ (see Fig. 2) reducing overall EW response. The top Teflon AF layer also influences the offset voltage. For instance, the offset voltage becomes $\Delta U=15 \mathrm{~V}$ for $d_{T}=50 \mathrm{~nm}$, and $\Delta U=6 \mathrm{~V}$ for $d_{T}=320 \mathrm{~nm}$. For constant PVDF-HFP thickness if Teflon AF thickness is increased, the ferroelectric contribution in the overall bilayer dielectric is also reduced. Hence, reduction in the offset voltage is observed. Thus, hysteretic contribution in dc-voltage EW can be controlled by the PVDF-HFP thickness, Teflon AF thickness and the applied voltage value. The synergy for efficient/effective EW response with low offset voltage amplitude can be maintained for thin layer of PVDF-HFP with Teflon AF thickness $d_{T} \sim 50 \mathrm{~nm}$.

Finally, it is important in application perspective to provide a test for durability of the (PVDF-HFP)/Teflon AF bilayer against degradation or delamination. Even for the continual exposure of the PVDF-HFP/ Teflon AF bilayer to the silicone oil environment for more than one week, no obvious degradation of the bilayer dielectric or delamination of the film was

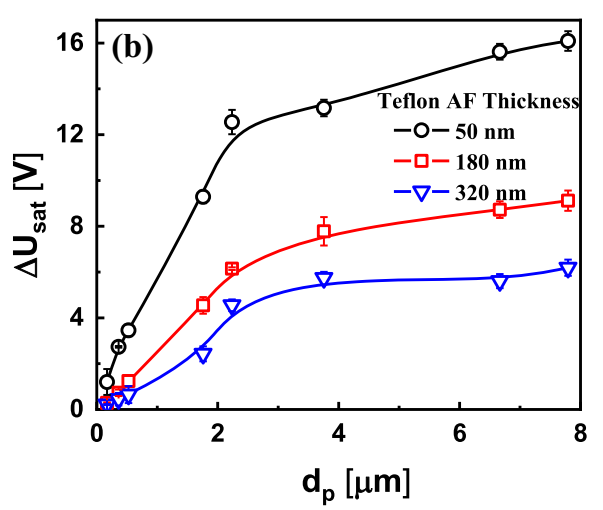

shows variation in $\Delta U$ versus $U_{\text {Max }}$ value. $\mathbf{b}$ The variation in offset voltage with increasing PVDF-HFP thicknesses for three values of Teflon AF thickness. 
observed. The durability of the bilayer was further tested by subjecting it to the electrowetting cycles under varying ambient temperature from -25 to $70{ }^{\circ} \mathrm{C}$ in silicone oil ambient. This EW response was tested on home-built ambient controlled cell [38]. The thermostable aqueous-ethylene glycol (EG) droplet was used as conductive drop phase to avoid the freezing of the saline water drop and silicone oil was an immiscible ambient phase. Figure 5 shows acvoltage EW response, plotted as $\Delta \cos \theta(U)$ against applied voltage square for temperatures varying from -25 to $70{ }^{\circ} \mathrm{C}$.

The thermostable aqueous-EG droplet on PVDF$\mathrm{HFP} / \mathrm{Teflon}$ AF bilayer demonstrates excellent EW response for temperature ranging from -25 to $70{ }^{\circ} \mathrm{C}$. The bilayer dielectric sustains this temperature variation without any failure/degradataion/delamination indicating its potential for ac-voltage EW-based outdoor devices and display panels.

\section{Conclusion}

In summary, nano-metre thin bilayer exhibits a maximum capacitance; hence, effective EW response is possible at low applied voltage. The bilayer, PVDFHFP $\left(d_{P} \sim 500 \mathrm{~nm}\right)$ and Teflon $\operatorname{AF}\left(d_{T} \sim 50 \mathrm{~nm}\right)$ exhibits maximum effective dielectric constant $\varepsilon_{e f f} \approx$ 8 that yields EW contact angle change of about $3.5^{\circ}$ per volt. Further decrease in thickness shows high capacitance but increase in leakage current leading to the early dielectric breakdown. EW response for dcvoltage exhibits hysteresis and the resultant offset

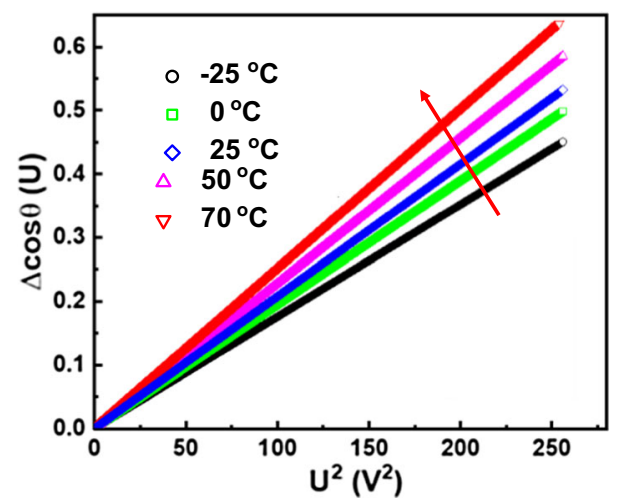

Figure 5 The plot of ac-voltage $\mathrm{EW}$ response, $\Delta \cos \theta(U)$ against voltage square $\left(U^{2}\right)$. PVDF-HFP/Teflon AF bilayer with $d_{P}=500 \mathrm{~nm}$ and top $d_{T}=50 \mathrm{~nm}$ was in silicone oil bath and subjected to temperatures variation from -25 to $70{ }^{\circ} \mathrm{C}$ indicated by the red arrow. voltage depends on amplitude of applied voltage and thickness of the polymer layers. This hysteresis found minimal for thin layers of PVDF-HFP and Teflon AF. The EW response on the bilayer over the wide temperature range from -25 to $70{ }^{\circ} \mathrm{C}$ indicates the stability of the dielectric layer over a broad range of ambient temperature. This demonstrates the high potential of the bilayer in EW-based applications. The polymer process parameters are simple and accessible for large area deposition make the bilayer as an immediate choice of the dielectric for EW-based experiments and suitable to cyber manufacturing ecosystem.

\section{Acknowledgements}

AGB gratefully acknowledge financial support under the research grant EMR/2016/007060, from the Science and Engineering Research Board (SERB), Govt. of India. SMW would like to acknowledge CSIR, Govt. of India, for senior research fellowship (SRF) fellowship (09/137(0578/2018-EMR-I). The technical assistance from Daniel Wijnperle, PCF, University of Twente, The Netherlands, for characterization of film thickness is greatly acknowledged.

\section{Authors contribution}

SMW: Methodology, Validation, Investigation, Data curation, Writing-original draft. YBS: Methodology, Writing-review \& editing. AVL: Writing—review \& editing. AGB: Conceptualization, Data curation, Writing -review \& editing, Supervision.

\section{Declarartions}

Conflict of interest The authors declare that they have no known competing financial interests or personal relationships that could have appeared to influence the work reported in this paper.

Supplementary Information: The online version contains supplementary material available at http s://doi.org/10.1007/s10853-021-06308-z. 


\section{References}

[1] Li J, Kim CJ (2020) Current commercialization status of electrowetting-on-dielectric (ewod) digital microfluidics. Lab Chip 20:1705-1712

[2] Mugele F, Baret JC (2005) Electrowetting: from basics to applications. J Phys-Condens Mat 17:R705-R774

[3] Berge B, Peseux J (2000) Variable focal lens controlled by an external voltage: an application of electrowetting. Eur Phys J E 3:159-163

[4] Mishra K, Murade C, Carreel B et al (2014) Optofluidic lens with tunable focal length and asphericity. Sci Rep-Uk. h ttps://doi.org/10.1038/srep06378

[5] Heikenfeld J, Zhou K, Kreit E et al (2009) Electrofluidic displays using young-laplace transposition of brilliant pigment dispersions. Nat Photonics 3:292-296

[6] Lee PTC, Chiu CW, Lee TM et al (2013) First fabrication of electrowetting display by using pigment-in-oil driving pixels. Acs Appl Mater Inter 5:5914-5920

[7] Zohrabi M, Lim WY, Cormack RH, Supekar OD, Bright VM, Gopinath JT (2019) Lidar system with nonmechanical electrowetting-based wide-angle beam steering. Opt Express 27:4404-4415

[8] Eaker CB, Joshipura ID, Maxwell LR, Heikenfeld J, Dickey MD (2017) Electrowetting without external voltage using paint-on electrodes. Lab Chip 17:1069-1075

[9] Haller B, Gopfrich K, Schroter M, Janiesch JW, Platzman I, Spatz JP (2018) Charge-controlled microfluidic formation of lipid-based single- and multicompartment systems. Lab Chip 18:2665-2674

[10] Satoh W, Hosono H, Suzuki H (2005) On-chip microfluidic transport and mixing using electrowetting and incorporation of sensing functions. Anal Chem 77:6857-6863

[11] Latip ENA, Coudron L, McDonnell MB et al (2017) Protein droplet actuation on superhydrophobic surfaces: a new approach toward anti-biofouling electrowetting systems. Rsc Adv 7:49633-49648

[12] Jain V, Muralidhar K (2020) Electrowetting-on-dielectric system for covid-19 testing. Trans Indian Natl Acad Eng 5:251-254

[13] Huang X, Liang C-C, Li J, Ho T-Y, Kim C-J (2019) Opensource incubation ecosystem for digital microfluidics - status and roadmap: Invited paper. In: IEEE/ACM International conference on computer-aided design, pp 1-6

[14] Ibrahim M, Chakrabarty K (2017) Cyber-physical digitalmicrofluidic biochips: Bridging the gap between microfluidics and microbiology. In: Proceedings of the IEEE, vol 106, issue 9, pp 1717-1743

[15] Berge B (1993) Electrocapillarité et mouillage de films isolants par l'eau. C R Acad Sci Paris III 317:63
[16] Banpurkar AG, Sawane Y, Wadhai SM et al (2017) Spontaneous electrification of fluoropolymer-water interfaces probed by electrowetting. Faraday Discuss 199:29-47

[17] Koo B, Kim CJ (2013) Evaluation of repeated electrowetting on three different fluoropolymer top coatings. J Micromech Microeng 23(6):067002

[18] Wan ZL, Zeng HJ, Feinerman A (2006) Area-tunable micromirror based on electrowetting actuation of liquidmetal droplets. Appl Phys Lett 89(20):201107

[19] Lippmann G (1875) Relation entre les phénomènes électriques et capillaires. Ann Chim Phys 5:494-549

[20] Wang J, Jin HX, Zhang JW et al (2018) The preparation of urchin-like La2O3 film and the superlow voltage electrowetting-on-dielectric response. Mater Lett 233:207-210

[21] Khan I, Castelletto S, Rosengarten G (2018) Deposition method and performance of $\mathrm{siO} 2$ as a dielectric material for beam steering electrowetting devices. Mater Res Express 5(7):076304

[22] Hou JX, Feng YC, Liao JL et al (2018) Multiscale interface effect on homogeneous dielectric structure of $\mathrm{ZrO} 2 /$ Teflon nanocomposite for electrowetting application. Polymers Basel 10(10):1119

[23] Berry S, Kedzierski J, Abedian B (2007) Irreversible electrowetting on thin fluoropolymer films. Langmuir 23:12429-12435

[24] Huang LX, Koo B, Kim CJ (2013) Sputtered-anodized $\mathrm{Ta} 2 \mathrm{O} 5$ as the dielectric layer for electrowetting-on-dielectric. J Microelectromech S 22:253-255

[25] Jin HX, Wang J, Yin YY et al (2017) The preparation of La2O3@AAO with simple hydrothermal method under ambient pressure and the enhanced electrowetting-on-dielectric performance. Superlattice Microst 110:233-242

[26] Sohail S, Mistri EA, Khan A, Banerjee S, Biswas K (2016) Fabrication and performance study of BST/Teflon nanocomposite thin film for low voltage electrowetting devices. Sensor Actuat Phys 238:122-132

[27] Papageorgiou DP, Tserepi A, Boudouvis AG, Papathanasiou AG (2012) Superior performance of multilayered fluoropolymer films in low voltage electrowetting. J Colloid Interf Sci 368:592-598

[28] Kilaru MK, Heikenfeld J, Lin G, Mark JE (2007) Strong charge trapping and bistable electrowetting on nanocomposite fluoropolymer: BaTiO3 dielectrics. Appl Phys Lett 90(21):212906

[29] Bune AV, Fridkin VM, Verkhovskaya KA, Taylor GW (1989) Photoelectric properties of the ferroelectric polymer PVDF. Ferroelectrics 99:203-212

[30] Kepler RG, Anderson RA (1992) Ferroelectric polymers. Adv Phys 41:1-57 
[31] Sawane YB, Ogale SB, Banpurkar AG (2016) Low voltage electrowetting on ferroelectric PVDF-HFP insulator with highly tunable contact angle range. ACS Appl Mater Inter 8:24049-24056

[32] Yedewar PG, Wadhai SW, Banpurkar AG (2020) Ac and dc voltage electrowetting on ferroelectric polymer for low voltage applications. AIP Conf Proc 2265:030309

[33] Markodimitrakis IE, Sema DG, Chamakos NT, Papadopoulos P, Papathanasiou AG (2021) Impact of substrate elasticity on contact angle saturation in electrowetting. Soft Matter 17:4335-4341

[34] Mugele F (2009) Fundamental challenges in electrowetting: From equilibrium shapes to contact angle saturation and drop dynamics. Soft Matter 5:3377-3384
[35] Banpurkar AG, Nichols KP, Mugele F (2008) Electrowetting-based microdrop tensiometer. Langmuir 24:10549-10551

[36] Bhushan B, Pan YL (2011) Role of electric field on surface wetting of polystyrene surface. Langmuir 27:9425-9429

[37] Yuan D, Li ZB, Thitsartarn W et al (2015) Beta phase PVDFHFP induced by mesoporous sio2 nanorods: synthesis and formation mechanism. J Mater Chem C 3:3708-3713

[38] Wadhai SM, Sawane YB, Banpurkar AG (2020) Electrowetting behaviour of thermostable liquid over wide temperature range. J Mater Sci 55:2365-2371. https://doi.org/10. 1007/s10853-019-04120-4

Publisher's Note Springer Nature remains neutral with regard to jurisdictional claims in published maps and institutional affiliations. 